\section{Estado nutricional materno, ganho de peso gestacional e peso ao nascer}

\section{Maternal nutritional status, gestational weight gain and birth weight}

\author{
Adriana Suely de Oliveira Melo' \\ Paula Lisiane Assunção' \\ Sheila Sherezaide Rocha Gondim ${ }^{1}$ \\ Danielle Franklin de Carvalho' \\ Melania Maria Ramos Amorim ${ }^{2}$ \\ Maria Helena D'Aquino Benicio ${ }^{3}$ \\ Maria Aparecida Alves Cardoso' \\ ${ }^{1}$ Núcleo de Estudos e Pesquisas Epidemiológicas - NEPE/Universidade Estadual \\ da Paraíba (UEPB) \\ ${ }^{2}$ Instituto Materno Infantil Professor Fernando Figueira - IMIP/PE \\ ${ }^{3}$ Departamento de Nutrição/NUPENS/USP
}

\section{Resumo}

Introdução: Tanto o estado nutricional materno como o ganho de peso gestacional vem sendo estudado em relação ao papel determinante que desempenham sobre o crescimento fetal e o peso ao nascer. O peso inadequado ao nascer é uma das grandes preocupações da saúde pública devido ao aumento da morbimortalidade no primeiro ano de vida e ao maior risco de desenvolver doenças na vida adulta, tais como a síndrome metabólica, nos casos de baixo peso, e diabetes e obesidade, nos casos de macrossomia. O objetivo deste trabalho foi descrever uma coorte de gestantes, classificando-as de acordo com o estado nutricional inicial, o ganho ponderal gestacional, a resistência nas artérias uterinas e o peso dos recém-nascidos. Métodos: foi acompanhada, a cada quatro semanas gestacionais, uma coorte de 115 gestantes atendidas pelo Programa de Saúde da Família do município de Campina Grande, PB. O estado nutricional inicial foi determinado através do índice de massa corporal $\left(\mathrm{kg} / \mathrm{m}^{2}\right)$ para a idade gestacional, e as gestantes classificadas de acordo com os critérios de Atalah. Na $20^{\mathrm{a}}$ semana, foi estudada a resistência das artérias uterinas, através da dopplervelocimetria. Resultados: o estado nutricional inicial mostrou uma alta prevalência de sobrepeso e obesidade (27\%), e uma prevalência significante de desnutrição (23\%). Um alto percentual de gestantes ganhou peso excessivo tanto no segundo (44\%) como no terceiro trimestre (45\%). A distribuição do peso ao nascer, indicou uma incidência de $10 \%$ de baixo peso e de $9 \%$ de macrossomia. Observou-se ainda, uma alta prevalência de incisuras nas artérias uterinas.

Palavras-chave: Peso ao nascer. Ganho de peso gestacional. Gravidez. Artérias uterinas.

Financiamento: CNPq - Edital CT-Saúde. № 30/2004 - Processo 505912/2004-0 
Abstract

Introduction: Maternal nutritional status and gestational weight gain have been addressed because of their importance to fetal growth and birth weight. Inadequate birth weight is a major concern to public health given it has been associated with increasing morbidity-mortality during the first year of life and with increased risks of chronic diseases in adult life such as metabolic syndrome for cases of low birth weight and diabetes and obesity for macrossomic infants. The objective of this study was to describe a cohort of pregnant women according to their nutritional status, gestational weight gain, uterine artery notches, and birth weight. Methods: A cohort of 115 pregnant women attending the Family Health Program in Campina Grande, PB, was evaluated every four gestational weeks. The initial maternal nutritional status was determined through the body mass index $\left(\mathrm{kg} / \mathrm{m}^{2}\right)$ and women were classified according to Atalah's criteria for gestational age. Arterial resistance was assessed through Doppler velocimetry on the $20^{\text {th }}$ week of pregnancy. Results: overweight and obesity were observed in $27 \%$ of the sample whereas $23 \%$ were undernourished. A high incidence of excessive weight gain was found in the second (44\%) and in the third quarter (45\%). Birth weight distribution indicated that $10 \%$ were of low birth weight and there were $9 \%$ of macrossomic babies. A high prevalence of uterine artery diastolic notches of was observed.

Keywords: Birth weight. Gestational weight gain. Pregnancy. Uterine arteries.

\section{Introdução}

O estado nutricional materno, assim como o ganho de peso gestacional, vem sendo foco atual de vários estudos, não apenas pela crescente prevalência dos seus distúrbios, mas sobretudo devido ao seu papel determinante sobre os desfechos gestacionais. Dentre estes destacam-se o crescimento fetal e o peso ao nascer, que podem trazer implicações para a saúde do indivíduo ao longo de sua vida, particularmente, em relação às doenças crônicas não tramissíveis ${ }^{1,2}$.

O estado nutricional é determinado, principalmente, pela ingestão de nutrientes, seja em termos de micro ou macronutrientes; portanto, um inadequado aporte energético da gestante pode levar a uma competição entre a mãe e o feto, limitando a disponibilidade dos nutrientes necessários ao adequado crescimento fetal ${ }^{3,4}$.

O período da gestação em que o ganho de peso materno tem uma maior influência sobre o crescimento fetal ainda é controverso $^{5,6}$. No entanto, o conhecimento desta relação é de extrema importância para a saúde pública, uma vez que desvios da normalidade podem ser controlados através de uma adequada assistência prénatal.

É importante observar que o crescimento fetal sofre também influência de fatores genéticos, do potencial biológico e de vários fatores reguladores e moduladores, como os ambientais, fetais e placentários ${ }^{3}$.

Somando-se aos fatores descritos acima, merece ainda destaque o fato de que o crescimento fetal está diretamente relacionado ao desenvolvimento placentário e à sua capacidade funcional.

O desenvolvimento placentário adequado depende do aumento do fluxo sangüíneo através das artérias uterinas. Este aumento do fluxo é determinado pela migração e invasão do citotrofoblasto extravilositário em direção às arteríolas espiraladas, com degeneração do endotélio vascular e perda da camada músculo-elástica, tornando-as mais distensíveis, 
com conseqüente redução da resistência destes vasos ${ }^{7}$.

Essas modificações no fluxo das artérias uterinas refletem-se na dopplervelocimetria, através da redução no índice de resistência com o evoluir da gestação ${ }^{8}$. Quando este processo se dá de forma incompleta, o resultado são vasos de alta resistência, podendo influenciar, de maneira decisiva, o crescimento fetal. A presença de incisura associada ao Índice de Pulsatilidade (IP) $\geq 1,45$ traduz a ocorrência de vasos de alta resistência e risco de insuficiência placentária ${ }^{9}$.

Acerca do tema exposto observa-se que estudos envolvendo o estado nutricional materno, a evolução ponderal durante a gestação e o peso ao nascer são freqüentes; entretanto, ainda são escassos os trabalhos que levam também em consideração a função placentária. Nenhum artigo foi encontrado nos bancos de dados Medline, Lilacs/sciELO e a Biblioteca Cochrane que abordasse o tema através da ótica da dopplervelocimetria (resistência das artérias uterinas).

O objetivo deste estudo é descrever as características de uma coorte de gestantes, classificando-as de acordo com o estado nutricional inicial, o ganho ponderal gestacional e a resistência nas artérias uterinas, em busca de subsídios que justifiquem estudos futuros mais aprofundados, avaliando a influência destes fatores associados à gênese do peso inadequado ao nascer.

\section{Métodos}

Trata-se de um estudo do tipo longitudinal, desenvolvido dentro do Projeto "Impacto da atividade física e da orientação alimentar, durante a gestação, sobre o ganho de peso gestacional e desfechos da gravidez", coordenado pelo Núcleo de Pesquisa em Nutrição e Saúde da Universidade de São Paulo (NUPENS/USP). Para este artigo foram utilizados dados de 115 gestantes que iniciaram pré-natal no Programa Saúde da Família (PSF) do município de Campina Grande-PB. As gestantes foram acompanhadas até o parto e o processo de coleta e o seguimento durou um ano (março de 2005 a março de 2006). O projeto desta pesquisa foi aprovado pelo Comitê de Ética da Universidade Estadual da Paraíba.

A amostra de conveniência estudada é capaz de detectar coeficientes de correlação de "0,25 ou maior" com poder de teste de $80 \%$ e um alfa de 0,05 . É capaz também de detectar prevalências de até $30 \%$, com precisão absoluta de oito pontos percentuais e intervalo de confiança igual a 95\%.

Foram incluídas gestantes com idade igual ou superior a 18 anos, com idade gestacional menor ou igual a 16 semanas (confirmada através de ultrassonografia), gestação única e feto vivo. As gestantes foram convidadas a participar do estudo pela equipe do PSF, sendo encaminhadas para a equipe do projeto. Depois de prestadas as informações sobre o estudo, caso aceitassem participar, assinavam o Termo de Consentimento Livre e Esclarecido. Foram excluídas gestantes com as seguintes condições: doenças maternas crônicas, ruptura prematura das membranas, parto antes da $36^{\text {a }}$ semana, anomalias ou perdas fetais.

A partir da $16^{\text {a }}$ semana gestacional, a cada quatro semanas as gestantes eram entrevistadas por graduandos da área de saúde, devidamente treinados. Na ocasião, era realizado um exame ultrassonográfico e as gestantes eram pesadas, usando batas de TNT (tecido não tecido) e descalças (balança digital Tanita). Os questionários, elaborados para o estudo e pré-testados através de estudo piloto, abordavam informações obstétricas, socioeconômicas e clínicas. A altura foi aferida no primeiro encontro, utilizando-se antropômetro móvel, com acuidade de um centímetro.

Para a classificação do estado nutricional inicial das mulheres em baixo peso, eutrofia, sobrepeso e obesidade, foram considerados os níveis críticos de Índice de Massa Corporal (IMC) para a idade gestacional, propostos por Atalah et al. ${ }^{10}$, e adotados pelo Ministério da Saúde. 
O ganho ponderal, no segundo trimestre, foi calculado através da diferença entre o peso materno na $28^{\mathrm{a}}$ e $16^{\mathrm{a}}$ semana e, no terceiro trimestre, através da diferença entre o peso materno na $36^{\mathrm{a}}$ e $28^{\mathrm{a}}$ semana. Para classificar a adequação do ganho de peso gestacional, no segundo e no terceiro trimestres, foram utilizados os critérios do Institute of Medicine (IOM) ${ }^{11}$, que estabelece limites para o ganho de peso gestacional de acordo com o estado nutricional inicial da gestante. As gestantes foram classificadas nas seguintes categorias: ganho de peso insuficiente, adequado ou excessivo.

Estimou-se o risco de insuficiência placentária a partir do estudo das artérias uterinas, na 20a semana, através de dopplervelocimetria. A ocorrência de incisura bilateral nas artérias uterinas, na presença de IP maior ou igual a 1,45 , foi utilizada como critério preditor para insuficiência placentária.

Os exames ultrassonográficos (dopplervelocimetria) foram realizados em aparelho Schimadzu (SSD-2200), usando-se sonda convexa de 3,75 mHZ, normalmente adotada como padrão para exames obstétricos. Um único observador, especialista em medicina fetal, efetuou todos esses exames.

Coletaram-se dados referentes aos recém-nascidos (RNs) nos prontuários das maternidades. O peso ao nascer foi classificado segundo os critérios da Organização Mundial da Saúde (OMS) ${ }^{12}$ em: baixo peso (RN com menos de $2.500 \mathrm{~g}$ ), peso insuficiente (RN com peso entre 2.500 e 2.999 g), peso adequado (RN com peso entre 3.000 e 3.999 g) e excesso de peso ou macrossomia (RN com 4.000 g ou mais).

\section{Resultados}

Foram captadas 137 gestantes. Destas, 22 foram consideradas perdas (11 desistências, quatro abortamentos, dois casos de amniorrexe prematura, quatro partos antes da $36^{\text {a }}$ semana e um caso de malformação fetal), resultando em 115 gestantes.
A idade média foi de $24 \pm 5$ anos, sendo que as mais jovens (24\%) tinham idade entre 18 e 20 anos $\left(\mathrm{IC}_{95 \%}: 17-33\right)$ e $3 \%\left(\mathrm{IC}_{95 \%}\right.$ : 1 - 9) estavam na faixa etária superior a 35 anos. A média dos anos de estudo das gestantes foi igual a $7 \pm 3$, sendo que $64 \%$ delas estudaram menos de oito anos.

Cerca de um terço das gestantes (35\%) trabalhava fora de casa $\left(\mathrm{IC}_{95 \%}: 27-45\right)$. Noventa por cento das mulheres tinham renda familiar per capita abaixo de um salário mínimo $\left(\mathrm{IC}_{95 \%}: 85\right.$ - 96).

Observou-se que $9 \%\left(\mathrm{IC}_{95 \%}: 4-15\right)$ das mulheres permaneciam fumando durante a gestação e $11 \%$ afirmaram ter usado maconha anteriormente, entretanto somente uma permanecia fazendo uso da droga durante a gestação. Apenas uma gestante praticava esporte.

A média do intervalo interpartal foi de $3,8 \pm 2,7$ anos, sendo que $54 \%$ (IC $_{95 \%}: 42-$ 65) das gestantes apresentaram um espaçamento entre 2 e 5 anos. Trinta e quatro por cento $\left(\mathrm{IC}_{95 \%}: 25\right.$ - 43) das gestantes estavam na primeira gestação e, entre as multíparas, $18 \%\left(\mathrm{IC}_{95 \%}: 14-22\right)$ tinham mudado de companheiro desde a última gestação.

O número médio de consultas pré-natais realizadas no PSF foi de $7 \pm 1,8$, sendo que $87 \%\left(\mathrm{IC}_{95 \%}: 79-92\right)$ das gestantes realizaram mais de seis consultas. $\mathrm{O}$ início do pré-natal ocorreu com a idade gestacional média de $10 \pm 3,2$ semanas.

A avaliação do estado nutricional inicial materno mostrou que metade das gestantes eram eutróficas e, como demonstra a Figura 1, o restante da distribuição ficou dividido entre o excesso de peso e a desnutrição. O peso materno com 16 semanas variou de 41,2 a 99,4 kg, com peso médio de 60,3 $\pm 11,0 \mathrm{~kg}$.

A média do ganho de peso total foi de 10,3 $\pm 3,6$, com ganho mínimo de 2,8 kg e máximo de 20,8 kg (Tabela 1).

Mais da metade das gestantes apresentou incisura nas duas artérias uterinas na $20^{a}$ semana de gestação. A presença de incisura bilateral associada ao IP $\geq 1,45$ mostrou que $10 \%$ do total dos fetos esta- 


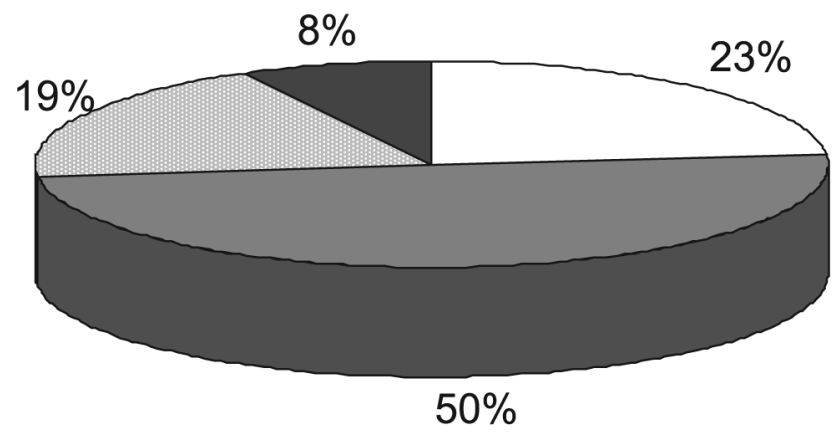

$\square$ Baixo peso $\square$ Peso adequado $\square$ Sobrepeso $\square$ Obesidade

Figura 1 - Distribuição percentual das gestantes segundo o estado nutricional inicial. Campina Grande-PB, 2006.

Figure 1 - Percentage distribution of pregnant women according to initial nutritional status. Campina Grande/PB, 2006.

Tabela 1 - Distribuição das gestantes segundo o ganho ponderal no segundo e no terceiro trimestres. Campina Grande - PB, 2006.

Table 1 - Distribution of pregnant women according to weight gain in the second and third quarters. Campina Grande/PB, 2006.

\begin{tabular}{llll}
\hline Variáveis & $\mathrm{N}$ & $\%$ & IC 95\% \\
\hline Ganho ponderal no segundo trimestre & & & \\
$\quad$ Insuficiente & 13 & 12 & $6-18$ \\
$\quad$ Adequado & 51 & 44 & $35-54$ \\
$\quad$ Excessivo & 51 & 44 & $35-54$ \\
\hline Ganho ponderal no terceiro trimestre & & & \\
$\quad$ Insuficiente & 26 & 22 & $15-31$ \\
$\quad$ Adequado & 37 & 32 & $24-41$ \\
$\quad$ Excessivo & 52 & 45 & $36-55$ \\
\hline
\end{tabular}

vam sob risco de crescimento insuficiente (Tabela 2).

A distribuição dos RNs, de acordo com a classificação do peso ao nascer e recomendações da OMS, indica que cerca de um terço das crianças apresentaram-se fora da normalidade (Figura 2). A incidência de crianças com baixo peso, quando somada à de peso insuficiente $(2.500 \mathrm{e}$ $2.999 \mathrm{~g}$ ), acumula um percentual de $24 \%$ $\left(\mathrm{IC}_{95 \%}: 17-33\right)$ de RNs com peso abaixo do ideal (3.000 e $3.999 \mathrm{~g})$.

Um maior percentual de crianças com baixo peso ao nascer (BPN) e peso insuficiente (PIN) foi observado entre as gestantes desnutridas, mostrando uma associa- ção estatisticamente significante $(\mathrm{p}=0,005)$ (Tabela 3).

\section{Discussão}

A classificação das gestantes, segundo o estado nutricional no início da gestação, indicou uma prevalência de sobrepeso e obesidade $(27 \%)$ semelhante aos observados por Nucci et al. ${ }^{13}$ (28\%) em gestantes que receberam assistência pré-natal no Sistema Único de Saúde, em seis capitais brasileiras (incluindo duas capitais nordestinas). Prevalências menores foram descritas por Lima e Sampaio ${ }^{14}$, em estudo realizado no Piauí, e por Aquino ${ }^{15}$ no 
Tabela 2 - Distribuição das gestantes de acordo com a presença de incisura e do índice de pulsatilidade, nas artérias uterinas, na 20a semana. Campina Grande-PB, 2006.

Table 2 - Distribution of pregnant women according to the presence of a notch and pulsatility index in uterine arteries in the 20 th week. Campina Grande/PB, 2006.

\begin{tabular}{lllc}
\hline Variáveis & $\mathrm{n}$ & $\%$ & IC 95\% \\
\hline Presença de incisuras & & & \\
$\quad$ Bilateral & 59 & 51 & $42-61$ \\
$\quad$ Unilateral & 17 & 15 & $9-23$ \\
$\quad$ Ausente & 39 & 34 & $25-43$ \\
\hline Incisura bilateral ( $=59)$ & & & \\
IP $<1,45$ & 47 & 80 & $70-90$ \\
IP $\geq 1,45$ & 12 & 20 & $10-31$ \\
\hline
\end{tabular}

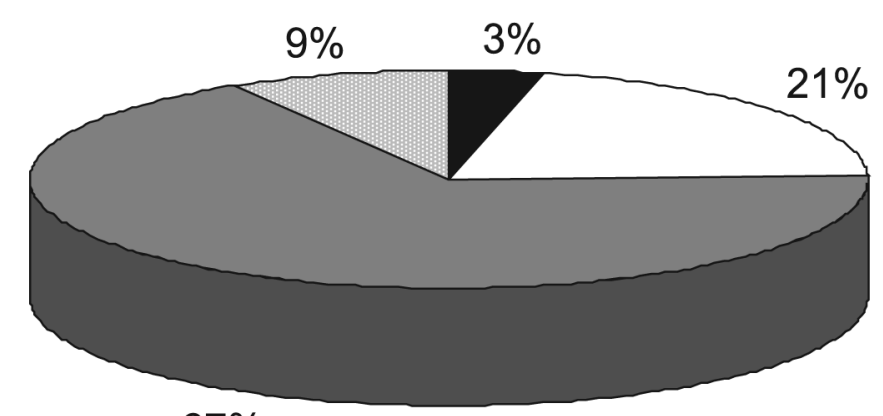

$67 \%$

Baixo peso $\square$ Peso insuficiente $\square$ Peso adequado $\square$ Macrossomia

Figura 2 - Distribuição percentual dos RNs de acordo com o peso ao nascer, segundo os critérios da OMS. Campina Grande-PB, 2006.

Figure 2 - Percentage distribution of neonates according to birth weight, according to WHO criteria. Campina Grande/PB, 2006.

Tabela 3 - Associação entre o estado nutricional materno no início da gestação e o baixo peso ao nascer e peso insuficiente. Campina Grande-PB, 2006.

Table 3 - Association between the nutritional status at beginning of pregnancy, and low birth weight and insufficient weight. Campina Grande/PB, 2006.

\begin{tabular}{lccccc}
\hline & \multicolumn{5}{c}{ BPN/PIN* } \\
\cline { 2 - 6 } Estado Nutricional Materno & $\mathrm{n}$ & Sim & $\%$ & $\mathrm{n}$ & $\%$ \\
\hline Desnutridas & 12 & 44 & 15 & 56 \\
Não Desnutridas & 16 & 18 & 72 & 80 \\
\hline
\end{tabular}

* PIN $=$ Peso insuficiente ao nascer

$\chi^{2}=7,67 ; p=0,005$ 
Distrito Federal (14,4\% e 17\%, respectivamente).

A prevalência da desnutrição na coorte foi consideravelmente alta (23\%), bem maior que aquela encontrada pelo estudo de Nucci et al. ${ }^{13}$ (6\%). No entanto, é importante ressaltar que, para a classificação do estado nutricional materno, estes autores utilizaram os critérios da OMS para a população adulta, que define como de baixo peso a gestante com IMC inferior a 18,5. Este valor é menor que o estabelecido por Atalah et al. ${ }^{10}$, utilizado como referência nesta pesquisa, devido ao fato de ser, atualmente, a curva adotada pelo Ministério da Saúde para o acompanhamento do ganho ponderal na gestação. Prevalências semelhantes de baixo peso materno foram observadas por Aquino ${ }^{15}$ (23\%) e por Sampaio $^{14}$ (18\%), utilizando a mesma metodologia do nosso estudo.

A incidência de ganho de peso excessivo nos dois trimestres estudados foi bem superior à observada em relação ao ganho de peso insuficiente. Estudos realizados em outras regiões brasileiras, com metodologia semelhante, indicaram percentuais de ganho ponderal excessivo mais baixos $^{15,16}$. A análise do ganho de peso gestacional em 240 gestantes de baixa renda da região metropolitana de Recife indicou que este aumentou de acordo com o estado nutricional inicial das gestantes, variando entre $31 \%$ para as mães desnutridas a $60,3 \%$, no caso das mulheres com sobrepeso/obesidade ${ }^{17}$.

Analisando os resultados dentro da atual perspectiva da transição nutricional do país, parece existir uma maior tendência à obesidade na Região Nordeste, mas apenas estudos posteriores poderão confirmar. Se, por um lado, encontra-se ainda uma prevalência elevada de desnutrição no início da gravidez, são, todavia, bastante elevadas as taxas de sobrepeso e obesidade, o que poderia ser explicado pela baixa escolaridade e pelos hábitos alimentares atuais que privilegiam uma dieta rica em carboidratos e lipídeos ${ }^{18,19}$. Ambos os fatores seriam um dos reflexos das respostas em cascata determinadas pela condição socioeconômica inferior, característica desta coorte.

Além disto, fatores culturais favorecem o ganho ponderal excessivo na gestação. Ainda é de senso comum que toda e qualquer gestante deve dobrar o seu aporte calórico e que o ganho de peso ideal deve ser em torno de 12 quilos, independente do estado nutricional inicial da gestante. Por fim, não devemos esquecer que, numa região onde a desnutrição sempre foi um grave problema de saúde, a obesidade ainda não é vista assim pela população em geral.

Os resultados observados para a incidência de incisura bilateral nas artérias uterinas chamaram a atenção neste estudo. Cerca de metade das gestantes da amostra $(51 \%)$ apresentaram incisura nas artérias uterinas, incidência bem mais alta do que aquelas relatados por estudos realizados em países desenvolvidos por Harrington e Campbell ${ }^{20}$, Albaiges et al..$^{9}$ e Papageorghiou et al. ${ }^{21}(3,9 \%, 5,1 \%$ e 9,3\%, respectivamente). Um estudo nacional, realizado por Costa et al..$^{22}$, relatou um percentual de $46,7 \%$ de incisura bilateral, bem próximo dos nossos resultados. É importante lembrar que os estudos nacionais foram realizados em gestantes de baixa renda, fato que poderia ser um sinalizador das diferenças observadas neste parâmetro para os países em desenvolvimento.

$\mathrm{O}$ alto percentual de primigestas, assim como um importante percentual de mulheres que mudaram de companheiro desde a gravidez anterior, entre as multíparas encontrados neste estudo, poderia ser uma das justificativas para esta alta prevalência de incisura bilateral, reforçando a hipótese de fatores imunológicos determinantes da resistência nas artérias uterinas. Além disso, não se pode afastar a possibilidade de fatores nutricionais interferindo na placentação, uma vez que tanto a etiologia da pré-eclâmpsia como da restrição de crescimento intra-uterino (CIUR) é complexa e multifatorial.

A baixa incidência do BPN (3,0\%) não é 
real, porque não computou as crianças nascidas com menos de 36 semanas $(n=7)$, um dos critérios de exclusão do estudo. Quando consideradas as exclusões, a incidência sobe para $10 \%$, aproximando-se da média nacional. Vale a pena salientar que, apesar da nossa média ser semelhante à nacional, talvez devêssemos esperar uma menor incidência, uma vez que o percentual do ganho ponderal excessivo foi bem maior do que a do insuficiente e as diferenças nos percentuais de desnutrição (23\%) e sobrepeso/obesidade (27\%) não foram tão marcantes.

Este questionamento em relação ao BPN levanta a hipótese de uma possível influência da alta incidência de incisura nas artérias uterinas observada na coorte, a qual estaria repercutindo sobre o crescimento fetal independente do estado nutricional materno e do ganho de peso gestacional. Esta hipótese seria reforçada pela presença de incisura bilateral verificada em todas as classificações de estado nutricional das gestantes (dados não apresentados) e um considerável percentual de BPN/PIN (18\%) entre as gestantes não desnutridas.

Se, por um lado, a média do baixo peso ao nascer não se mostrou fora do padrão brasileiro, chama a atenção a alta incidên- cia da macrossomia, acometendo $9 \%$ dos recém-nascidos, acima do esperado que seria de $5 \%{ }^{23,24}$. Uma das justificativas plausíveis para o alto percentual de RNs macrossômicos detectado nesta coorte foi a alta incidência de ganho de peso gestacional excessivo.

O número adequado de consultas e a grande quantidade de primigestas no estudo parece demonstrar a boa qualidade dos cuidados pré-natais oferecidos pelo Programa Saúde da Família local. Entretanto, as altas prevalências de sobrepeso/ obesidade entre as mulheres em idade reprodutiva e de ganho de peso excessivo observados neste estudo parecem refletir a transição nutricional em curso, ao mesmo tempo em que demonstram uma falta de controle do ganho de peso em mulheres em idade reprodutiva.

A definição de mais pesquisas que venham investigar a problemática do peso inadequado ao nascer, no sentido de buscar subsídios para prevenção da morbimortalidade infantil e adulta, ainda constitui uma necessidade em nosso país. $\mathrm{O}$ presente estudo abre perspectivas para futuras investigações que visem explorar as possíveis e intrincadas associações entres os diversos fatores determinantes do peso ao nascer.

\section{Referências}

1. Barker DJP, Osmond C, Golding J, Kuh D, Wadsworth MEJ. Growth in utero, blood pressure in childhood and adult life, and mortality from cardiovascular disease. BMJ 1989; 298: 564-71.

2. Zadik Z. Maternal nutrition, fetal weight, body composition and disease in later life. J Endocrinol Invest 2003; 26: 941-46.

3. Kramer MS. Determinants of low birth weight: methodological assessment and meta-analysis. Bull WHO 1987; 65: 666-737.

4. Kramer MS. Balanced protein/energy supplementation in pregnancy (Cochrane Review). Cochrane Library. Issue 4. Oxford: Update Software; 2002.
5. Thame M, Osmond C, Bennett F, Wilks R, Forrester T. Fetal growth is directly related to maternal anthropometry and placental volume. Eur J Clin Nutr 2004; 58: 894-900.

6. Neufeld LM, Haas JD, Grajéda R, Martorell R. Changes in maternal weight from the first to second trimester of pregnancy are associated with fetal growth and infant length at birth. Am J Clin Nutr 2004; 79: 646-52.

7. Brosens I, Robertson WB, Dixon HG. The physiological response of the vessels of the placental bed to normal pregnancy. J Pathol Bacteriol 1967; 93: 569-79.

8. Parretti E, Mealli F, Magrini A, Cioni R, Mecacci F, La Torre $\mathrm{P}$, et al. Cross-sectional and longitudinal evaluation of uterine artery Doppler velocimetry for the prediction of pre-eclampsia in normotensive women with specific risk factors. Ultrasound Obstet Gynecol. 2003; 22: 160-5. 
9. Albaiges G, Missfelder-Lobos H, Lees C, Parra M, Nicolaides KH. One-stage screening for pregnancy complications by color Doppler assessment of the uterine arteries at 23 weeks' gestation. Obstet Gynecol 2000; 96: $559-64$.

10. Atalah E, Castillo CL, Castro RS, Amparo Aldea P. Propuesta de un Nuevo estándar de evaluación nutritional de embarazadas. Rev Med Chile 1997; 125: 1429-36.

11. Institute of Medicine, Subcommittee on Nutritional Status and weight gain during pregnancy. Nutrition during pregnancy. Washington: National Academy Press; 1990.

12. World Health Organization. Physical status: the use and interpretation of anthropometry. Report. Geneva; 1995. (WHO-Technical Reports Series, 854).

13. Nucci LB, Schmidt MI, Duncan BB, Fuchs SC, Fleck ET, Santos Britto MM. Nutritional status of pregnant women: prevalence and associated pregnancy outcomes. Rev Saúde Pública 2001; 35: 502-7.

14. Lima, GSP; Sampaio, HAC. Influência de fatores obstétricos, socioeconômicos e nutricionais da gestante sobre o peso do recém-nascido: estudo realizado em uma maternidade em Teresina, Piauí. Rev Bras Saude Mater Infant 2004; 3: 253-61.

15. Aquino, KKNC. Determinantes do ganho ponderal excessivo em gestantes atendidas nos serviços públicos de pré-natal do Distrito Federal [dissertação de mestrado]. Brasília: Universidade de Brasília - UNB; 2004.

16. Stulbach TE. Determinantes do ganho ponderal excessivo durante a gestação em um serviço público de pré-natal de baixo risco [dissertação de Mestrado]. São Paulo:

Faculdade de Saúde Pública - Universidade de São Paulo; 2003.
17. Andreto LM, Souza AI, Figueiroa JN, Cabral-Filho JE. Fatores asociados ao ganho ponderal excesivo em gestantes atendidas em um serviço público de pré-natal na cidade de Recife, Pernambuco, Brasil. Cad Saúde Pública 2006; 22: 2401-09.

18. Monteiro CA, Mondini L, Souza ALM, Popkin BM. Da desnutrição para a obesidade: A transição nutricional no Brasil.In: Monteiro CA (org.). Velhos e Novos Males da Saúde no Brasil. São Paulo: Editora Hucitec; 2000. pp. 247-255.

19. Batista Filho M, Rissin A. A transição nutricional no Brasil: tendências regionais e temporais. Cad Saúde Pública 2003; 19: 181-91.

20. Harrington K; Campbell S. Doppler ultrasound in prenatal prediction and diagnosis. Curr Opin Obstet Gynecol 1992; 4: 264-72.

21. Papageorghiou AT, Yu CK, Bindra R, Pandis G, Nicolaides KH. Fetal Medicine Foundation Second Trimester Screening Group. Multicenter screening for preeclampsia and fetal growth restriction by transvaginal uterine artery Doppler at 23 weeks of gestation. Ultrasound Obstet Gynecol 2001; 18: 441-9.

22. Costa, Fabrício da Silva. Análise Dopplervelocimétrica das Artérias Uterinas de Mulheres Primigestas. Rev Bras Ginecol Obstet 2001; 23(7): 469-69.

23. Monteiro, CA, Benício, MHD’A, Ortiz LP. Tendência secular do peso ao nascer na cidade de São Paulo (19761998). Rev Saúde Pública 2000; 34: 26-40.

24. Kac G, Velásquez-Meléndez G. Ganho de peso gestacional e macrossomia em uma coorte de mães e filhos. J Pediatr 2005; 81: 47-53.

Recebido em: 11/10/06

Versão final reapresentada em: 05/03/07 Aprovado em: 23/03/07 